\title{
AUARA, EL COMPROMISO DE LA EMPRESA SOCIAL CON LOS ODS
}

\author{
Autor: Antonio Espinosa de los Monteros \\ hola@auara.org \\ CEO y cofundador de AUARA
}

\section{Resumen}

En este artículo AUARA, empresa social que comercializa agua mineral natural y destina el $100 \%$ de sus dividendos a llevar agua potable a países que no disponen de este recurso, vincula su actividad a los Objetivos de Desarrollo Sostenible (ODS) desde que inició su actividad, septiembre 2016, a la actualidad. La compañía ha logrado entrar en beneficios en 2018, reafirmando su modelo de impacto social.

Palabras clave: ODS; Agenda 2030; empresa social; sostenibilidad, agua.

\section{AUARA, a social enterprise commitment to SDG}

\author{
Abstract \\ In this article AUARA, a social company that sells natural mineral water \\ and allocates $100 \%$ of its dividends to bring drinking water to countries that do


not have this resource, links his activity to the Sustainable Development Goals (SDG) since it began its activity, September 2016, to the present. The company has achieved profits in 2018 , reaffirming its social impact model.

Keywords: SDG; Agenda 2030; social enterprise; sustainability; water.

Fecha de recepción: 18/11/2019.

Fecha de aceptación: 20/11/2019.

"Hemos llegado a un momento decisivo para la historia de la humanidad. Los pueblos del mundo nos han pedido que alumbremos el camino hacia un futuro prometedor y lleno de oportunidades. Y los estados miembros han respondido con la Agenda 2030 para el Desarrollo Sostenible”, con estas palabras Ban Ki-moon, entonces secretario general de Naciones Unidas, cargo que ocupó entre 2007-2017, anunciaba en la clausura la Asamblea General de la ONU, del 25 de septiembre de 2015, la aprobación de los 17 Objetivos de Desarrollo Sostenible (ODS) por parte de los estados miembros, que están marcando la agenda global para afrontar los desafíos globales que tenemos como Humanidad: lucha contra la pobreza y el hambre, disminución de la desigualdad, cambio climático -que ha derivado en emergencia climática-, fomento de la igualdad de género, de la educación o del acceso al agua limpia y saneamiento.

Hace cuatro años AUARA estaba en fase de concepción. Y hace cuatro años el mundo afrontaba los mismos problemas que en la actualidad, aunque parece que se han ido incrementando. El hambre afecta a 821 millones de personas y lleva tres años aumentando, según el El estado de la seguridad alimentaria y la nutrición en el mundo de la FAO (15 de julio de 2019). Más de 70 millones de personas $(70,8)$ se han visto obligadas a abandonar sus hogares por los conflictos armados o la persecución, según el Informe de ACNUR Tendencias Globales, desplazamiento forzado en 2018, que indica que 25,9 millones de personas son refugiadas y más de la mitad menores de edad. Además, en menos de dos años se ha pasado de hablar de cambio climático a emergencia climática y los científicos nos lo recuerdan. El IPCC, el Grupo Intergubernamental de Expertos en Cambio Climático, lanzaba una señal de alarma en su informe de 2018 para transitar hacia una economía descarbonizada si queríamos cumplir con el Acuerdo de París para frenar el cambio climático y limitar cuanto antes el calentamiento global a 1,5 grados, que podría alcanzarse entre 2030 y 2050 si las emisiones de CO2 continúan a este ritmo. 
En septiembre de 2016 nació AUARA. Justo en el primer aniversario de los ODS, pero cuando fundamos la empresa social -Pablo Urbano, director de Operaciones (CCO) y cofundador; Luis de Sande, director Financiero y cofundador, y yo como CEO y cofundador- no pensamos de inicio en cómo podíamos contribuir a la Agenda 2030, aunque ya lo estábamos haciendo, sin ser conscientes. Nuestro propósito era y es el de acabar con la mayor de las pobrezas, la falta de acceso a agua porque sin este recurso no hay posibilidad de desarrollo.

De este modo, nos constituimos como empresa social que destina el 100\% de sus dividendos a proyectos sociales para llevar agua potable a poblaciones que carecen de ella. Y para ello, tuvimos que mirar fuera de España porque todavía no existe esta figura jurídica y nos convertimos en la primera empresa social española certificada por el sello Social Enterprise Mark (Reino Unido) para garantizar en nuestro pacto de socios que no nos desviaríamos de nuestro compromiso fundacional.

AUARA significa en amhárico, idioma etíope, "tormenta de arena que se produce en desiertos como el de Afar, el lugar más cálido y seco del planeta”. Surgió con una clara y proactiva vocación social con la que invita a todo el mundo que la consume a convertir un acto cotidiano como beber agua, en un acto extraordinario. Y le pusimos ese nombre después de que me fuese como voluntario a Etiopía y participase en la construcción de un hospital junto a la ONG Amigos de Silva y me di cuenta que la causa principal de que la gente acudiera al hospital venía motivada por enfermedades vinculadas con la falta de acceso a agua potable.

Antes había participado como voluntario en Perú y Camboya, pero tras llegar de Etiopía me encontré con Pablo, que era amigo del colegio, y unimos el problema del agua con la empresa social y creamos AUARA. Yo soy arquitecto y Pablo ingeniero, pero necesitábamos a alguien que nos ayudara a poner en marcha el proyecto y apareció Luis.

Desde entonces no hemos parado de trabajar para cumplir con nuestro propósito, llevar agua a poblaciones que carecen de ella y facilitar su desarrollo. Recientemente, acabamos de demostrar que los proyectos de impacto social también son rentables: en nuestro tercer año de actividad (2018) hemos entrado en beneficios, alcanzado el equilibrio entre los ingresos y los costes. Era algo que no nos preocupaba, pero que traslada un mensaje a la sociedad de que la empresa social también puede ser rentable y contribuir a mejorar la vida de las personas.

Coincidiendo con el cuarto aniversario de los ODS, el pasado mes de septiembre, decidimos analizar nuestra contribución a los mismos. Y comprobamos, que además de impactar directamente en los referidos al ODS 1 Fin de la pobreza, al ODS 6 Agua Limpia y Saneamiento y al ODS 17 Alianzas, quien bebe AUARA está contribuyendo directamente con 15 de los 17 ODS. 
Compartimos, modestamente, la vinculación de nuestra actividad con la Agenda 2030 con la idea de que pueda servir de inspiración a otras empresas sociales, empresas u organizaciones para alinearse con los ODS.

ODS 1: Fin de la pobreza. La falta de acceso a agua potable es la pobreza más grande que existe porque sin agua no hay desarrollo posible. La ONU reconoció en 2010 que el acceso al agua potable y saneamiento son esenciales para la realización de todos los derechos humanos y desde AUARA tratamos de contribuir con los 50 proyectos de acceso a agua que hemos realizado en 15 países hasta la fecha.

ODS 2: Hambre cero. Si unimos que en el mundo hay 821 millones de personas que pasan hambre y que 3 de cada 10 personas carecen de acceso a servicios seguros de agua potable, según la $\mathrm{ONU}$, la lucha contra la pobreza y el hambre no se podrá cumplir sin que el agua entre en la ecuación del desarrollo sostenible.

El agua es el recurso natural que da sentido a la propiedad y al control de las tierras evitando entre otros problemas la emigración a ciudades que no hace en ocasiones más que trasladar de sitio el problema del hambre y la pobreza. La falta de agua en los cultivos es la principal causa de hambrunas en lugares en los que se practica una agricultura de subsistencia y cuando hay sequía tener acceso a agua para cultivar es un seguro de vida. En AUARA tratamos de fomentar la autosuficiencia alimentaria y la mejora de la nutrición con los proyectos que desarrollamos.

ODS 3: Salud y bienestar. El acceso a agua potable es el primer paso para asegurar la salud de las personas y contribuye a la disminución de las enfermedades generadas por el agua como cólera, fiebre tifoidea, meningitis, hepatitis o diarrea. Cada año, 3,5 millones de personas mueren en el mundo por no tener acceso a agua potable o por enfermedades relacionadas con el agua, según el World Water Council, que calcula que cerca de 1.000 niños fallecen cada día por las mismas causas. De este modo, la falta de agua se ha convertido en un arma devastadora que causa más mortalidad que la peor de las guerras.

A través de los 50 proyectos que hemos desarrollado en estos años hemos permitido que 28.411 personas tengan acceso a agua potable y saneamiento, lo que permite reducir hasta en un $80 \%$ las enfermedades con un impacto inmediato en la tasa de mortalidad infantil.

ODS 4: Educación de calidad. Cuando en una zona o poblado no hay acceso a agua, las madres y las niñas son las que tienen que recorrer varios kilómetros cada día para rellenar sus bidones en el río más cercano y llevarlos de vuelta a casa para el abastecimiento de la familia, lo que les impide asistir a la escuela y adquirir una formación que les permita mirar al futuro con esperanza. Por eso, tener acceso a agua permite que las niñas puedan estudiar.

ODS 5: Igualdad de género. Cuando se trata de ir a por agua, el género masculino no existe y las mujeres y las niñas son las responsables de ir a buscarla. Se estima que pueden emplear hasta 40.000 millones de horas en ir a por agua y la 
liberación de tiempo disponible ofrece un mayor tiempo de dedicación a los hijos, la posibilidad de ir a la escuela, la participación en nuevas actividades de la comunidad, la adquisición de habilidades sociales y, en definitiva, la promoción de la igualdad de derechos.

ODS 6: Agua limpia y saneamiento. De lo que nos podemos sentir más orgullosos en AUARA es de haber facilitado el acceso a agua potable y saneamiento a 28.411 personas desde nuestro lanzamiento en 2016. Somos ambiciosos y en 2020 queremos asegurar el agua potable a 50.000 personas, duplicando la cifra actual en poco más de un año.

Nuestros compromiso fundacional con el ODS 6 tuvo su recompensa, sin buscarla, al ser galardonados en la primera edición de los Reconocimientos go!ODS (octubre 2018), impulsados por la Red Española de Pacto Mundial y la Fundación Rafael del Pino.

ODS 7: Energía asequible y no contaminante. No impactamos directamente.

ODS 8: Trabajo decente y crecimiento económico. El agua es prosperidad. En el campo genera mayor rendimiento de los cultivos y su dotación a nivel de infraestructuras genera mejor calidad de vida en las viviendas, posibilidad de trabajo y desarrollo comunitario.

El impacto social de AUARA proviene por dos vías. Por una parte, la confianza de los consumidores en nuestra oferta -agua mineral, agua con gas y, próximamente, refrescos a través de Planet Drink- y de la colaboración con las empresas con las que llevamos a cabo los proyectos en terreno. Pero también se genera desde el equipo de AUARA, formado por más de 20 profesionales comprometidos con el impulso de una sociedad más próspera y no lo podíamos hacer de otro modo que desde la gestión responsable de personas que genera orgullo de pertenencia.

ODS 9: Industria, innovación e infraestructira. No impactamos directamente.

ODS 10: Reducción de las desigualdades. Como elemento básico para el desarrollo, el agua es el recurso desde el que se empieza a reducir las desigualdades. Desde nuestro lanzamiento, en septiembre de 2016, hemos puesto en marcha 50 proyectos en poblaciones en situación de pobreza extrema, pertenecientes a 15 países de África, Asia y América Central.

ODS 11: Ciudades y comunidades sostenibles. El acceso a un saneamiento seguro incide directamente en la salud y mejora de las condiciones de vida. Se estima que 6 de cada 10 personas, unos 4.500 millones de personas en todo el mundo, carecen de un saneamiento seguro, según la Organización Mundial de la Salud (OMS). Desde AUARA contribuimos a reducir esa cifra gracias a nuestros proyectos.

ODS 12: Producción y consumo responsables. La venta de botellas de agua es el vehículo que tenemos para poder llevar este recurso a personas que no la tienen. Cuando pusimos en marcha AUARA, después de analizar las diferentes opciones 
para comercializar agua, nos dimos cuenta que envasar el agua en material RPET $100 \%$ reciclado y $100 \%$ reciclable era la opción que menos impacto medioambiental tendría, convirtiéndonos en la primera empresa europea en apostar por envasar toda nuestra producción en este material. Desde entonces hemos reciclado más de 138 toneladas de plástico, que se han traducido en el ahorro de más de 226.000 litros de petróleo. Para 2020 pretendemos evitar el empleo de 10 millones de litros de petróleo en RPET y reutilizar el plástico de 90 millones de botellas.

ODS 13: Acción por el clima. Estamos comprometidos con minimizar el impacto ambiental de nuestro producto promoviendo envases más eficientes (RPET $100 \%$ reciclado y reciclable), botellas con un diseño que permite el transporte de un $20 \%$ más en cada palet o una flota comercial de vehículos híbridos. Apostamos por la economía circular para minimizar nuestro impacto medioambiental.

ODS 14: Vida submarina. Nos nutrimos de agua dulce y no salada, pero sabemos que la sostenibilidad de los océanos y recursos marinos es fundamental para nuestra supervivencia y el cuidado de la biodiversidad. Por eso, apostamos por no generar más plástico sino usar el que ya está para que no llegue al mar.

ODS 15: Vida de ecosistemas terrestres. La disponibilidad de agua promueve la sostenibilidad de las comunidades y su desarrollo, disminuye la degradación y desertificación de las tierras.

ODS 16: Paz, justicia e instituciones sólidas. Con motivo del Día Mundial del Agua en 2019, lanzamos una campaña de sensibilización con un mensaje impactante en sus botellas para simbolizar que el agua, o su ausencia, es un arma mortal que cada año acaba con la vida de 3,5 millones de personas, según el World Water Council. Así, la aportación de agua a las comunidades facilita a creación de instituciones eficaces, responsables e inclusivas.

ODS 17: Alianzas para lograr los objetivos. El 17 es el ODS estrella, sin él, el resto parecen incompletos. Sin darnos cuenta, hemos visto que este ODS da sentido a todo nuestro propósito, llevar agua a países que carecen de este recurso. Y lo hemos hecho posible gracias a la colaboración de las entidades no lucrativas y de las empresas, porque de otro modo no podríamos tener impacto social.

En la actualidad, AUARA colabora con 10 organizaciones para desarrollar sus proyectos: África Directo, Ayuda en Acción, Zerca y Lejos, Fundación Acción Geoda, Fundación Esperanza y Alegría, Fundación Kirira, Fundación Promoción Social, Sauce ONG, Ongawa y Manos Unidas. Cada uno de los 50 proyectos se han implementado de la misma manera, acompañando durante cinco años a las organizaciones españolas responsables de cada uno de ellos y que cuentan con un aliado de referencia sobre el terreno para garantizar su buen desarrollo.

Estos proyectos no se podrían llevar a cabo sin la confianza de las 75 empresas que confían en el agua de AUARA y a las que desarrollan proyectos propios como Calidad Pascual, Más Móvil, Ilunión, Ahorramás, Selecta, Room Mate, Veritas, 
Aveda, BBVA, Fundación Universitaria San Pablo-CEU, Coolook, El Ganso, Gaia Camp, Grupo Álava, Grupo Lalala, Grupo Larrumba e Isban.

Esta alianza ha permitido que generemos más de 21,6 millones de litros de agua en países en vías de desarrollo que han beneficiado a 28.411 personas.

Por cada litro de agua vendido, AUARA es capaz de generar cuatro litros de agua en países en vías de desarrollo mediante la construcción de pozos o la instalación de tanques de recogida de agua de lluvia. Sin duda, todos los que hacemos realidad AUARA nos sentimos orgullosos de mejorar la sociedad y de trabajar para seguir impulsando el impacto social. 